\title{
Hausdorff limits of Rolle leaves
}

\author{
JEAN-MARIE LION AND PATRICK SPEISSEGGER
}

Let $\mathcal{R}$ be an o-minimal expansion of the real field. We introduce a class of Hausdorff limits, the $T^{\infty}$-limits over $\mathcal{R}$, that do not in general fall under the scope of Marker and Steinhorn's definability-of-types theorem. We prove that if $\mathcal{R}$ admits analytic cell decomposition, then every $T^{\infty}$-limit over $\mathcal{R}$ is definable in the pfaffian closure of $\mathcal{R}$.

\section{Introduction}

We fix an o-minimal expansion $\mathcal{R}$ of the real field. In this paper, we study $T^{\infty}$-limits over $\mathcal{R}$ as defined in Section 1 below; they generalize the pfaffian limits over $\mathcal{R}$ introduced in [5, Section 4]. Pfaffian limits over $\mathcal{R}$ are definable in the pfaffian closure $\mathcal{P}(\mathcal{R})$ of $\mathcal{R}$ [7], by the variant of Marker and Steinhorn's definability-of-types theorem [6] found in van den Dries [1, Theorem 3.1] and [4, Theorem 1]. The $T^{\infty}$-limits over $\mathcal{R}$ considered here do not seem to fall under the scope of these theorems, as explained in Section 1 below. Nevertheless, $T^{\infty}$-limits were used by Lion and Rolin [3] to establish the o-minimality of the expansion of $\mathbb{R}_{\mathrm{an}}$ by all Rolle leaves over $\mathbb{R}_{\mathrm{an}}$ of codimension one.

To state our results, we work in the setting of [5, Introduction]; in particular, recall that a set $W \subseteq \mathbb{R}^{n}$ is a Rolle leaf over $\mathcal{R}$ if there exists a nested Rolle leaf $\left(W_{0}, \ldots, W_{k}\right)$ over $\mathcal{R}$ such that $W=W_{k}$.

First, we obtain the following generalization of [3, Théorème 1].

Proclaim (Theorem A) Let $\mathcal{N}(\mathcal{R})$ be the expansion of $\mathcal{R}$ by all Rolle leaves over $\mathcal{R}$.

(1) There is an o-minimal expansion $T^{\infty}(\mathcal{R})$ of $\mathcal{N}(\mathcal{R})$ in which every $T^{\infty}$-limit over $\mathcal{R}$ is definable.

(2) Let $M \subseteq \mathbb{R}^{n}$ be a bounded, definable $C^{2}$-manifold and $d$ be a definable and integrable nested distribution on $M$. Let $K \subseteq \mathbb{R}^{n}$ be a $T^{\infty}$-limit obtained from $d$. Then $\operatorname{dim} K \leq \operatorname{dim} d$. 
The question then arises how $T^{\infty}(\mathcal{R})$ relates to the pfaffian closure $\mathcal{P}(\mathcal{R})$ of $\mathcal{R}$. Indeed, we do not know in general if $T^{\infty}(\mathcal{R})$ is interdefinable with $\mathcal{N}(\mathcal{R})$ or $\mathcal{P}(\mathcal{R})$, or if $T^{\infty}\left(T^{\infty}(\mathcal{R})\right.$ ) is interdefinable with $T^{\infty}(\mathcal{R})$. Based on [5], we can answer such questions under an additional hypothesis:

Proclaim (Theorem B) Assume that $\mathcal{R}$ admits analytic cell decomposition.

(1) Every $T^{\infty}$-limit over $\mathcal{P}(\mathcal{R})$ is definable in $\mathcal{P}(\mathcal{R})$.

(2) The structures $T^{\infty}(\mathcal{R})$ and $\mathcal{P}(\mathcal{R})$ are interdefinable; in particular, $T^{\infty}(\mathcal{R})$ and $T^{\infty}\left(T^{\infty}(\mathcal{R})\right)$ are interdefinable.

We view the combination of Theorems $\mathrm{A}(2)$ and $\mathrm{B}(1)$ as a non-first order extension of [1, Theorem 3.1] and [4, Theorem 1].

Our proofs of these theorems rely heavily on terminology and notation introduced in [5, Introduction and Section 2]; we do not repeat the respective definitions here. We prove Theorem A in Section 3 below using the approach of [7], but based on a straightforward adaptation of some results of [5, Section 4] to $T^{\infty}$-limits carried out in Section 2 below. Theorem B then follows by adapting [5, Proposition 7.1] to $T^{\infty}$-limits and using [5, Proposition 10.4]; the details are given in Section 4.

\section{The definitions}

Let $M \subseteq \mathbb{R}^{n}$ be a bounded, definable $C^{2}$-manifold of dimension $m$. We adopt the terminology and results found in [5, Introduction and Section 2], and we let $d=\left(d_{0}, \ldots, d_{k}\right)$ be a definable and integrable nested distribution on $M$.

A sequence $\left(V_{\iota}\right)_{\iota \in \mathbb{N}}$ of integral manifolds of $d_{k}$ is a $T^{\infty}$-sequence of integral manifolds of $d$ if there are a core distribution $e=\left(e_{0}, \ldots, e_{l}\right)$ of $d$, a sequence $\left(W_{\iota}\right)$ of Rolle leaves of $e$ and a definable family $\mathcal{B}$ of closed integral manifolds of $d_{k-l}$ such that each $V_{\iota}$ is an admissible integral manifold of $d$ with core $W_{\iota}$ corresponding to $e$ and definable part in $\mathcal{B}$ corresponding to $W_{\iota}$, as defined in [5, Definition 4.1].

In this situation, we call $\left(W_{\iota}\right)$ the core sequence of the sequence $\left(V_{\iota}\right)$ corresponding to $e$ and $\mathcal{B}$ a definable part of the sequence $\left(V_{\iota}\right)$ corresponding to $\left(W_{\iota}\right)$.

Remarks (1) We think of the core sequence of $\left(V_{\iota}\right)$ as representing the "non-definable part" of $\left(V_{\iota}\right)$. If $W_{\iota}=W_{1}$ for all $\iota$, then $\left(V_{\iota}\right)$ is an admissible sequence of integral manifolds of $d$ as defined in [5, Definition 4.3]. 
(2) Let $\left(V_{\iota}\right)$ be a $T^{\infty}$-sequence of integral manifolds of $d$. Then there is a $T^{\infty}$-sequence $\left(U_{\iota}\right)$ of integral manifolds of $\left(d_{0}, \ldots, d_{k-1}\right)$ such that $V_{\iota} \subseteq U_{\iota}$ for $\iota \in \mathbb{N}$.

Let $\left(V_{\iota}\right)$ be a $T^{\infty}$-sequence of integral manifolds of $d$. If $\left(V_{\iota}\right)$ converges to $K$ in $\mathcal{K}_{n}$ (the space of all compact subsets of $\mathbb{R}^{n}$ equipped with the Hausdorff metric), we call $K$ a $T^{\infty}$-limit over $\mathcal{R}$. In this situation, we say that $K$ is obtained from $d$, and we put

$$
\operatorname{deg} K:=\min \{\operatorname{deg} f: K \text { is obtained from } f\} .
$$

Remarks (3) It is unknown whether the family of all Rolle leaves of $e$ is definable in $\mathcal{P}(\mathcal{R})^{1}$. As a consequence, contrary to the situation described by [5, Lemma 4.5] for pfaffian limits over $\mathcal{R}$, the variant of Marker and Steinhorn's definabilityof-types theorem [6] found in [1, Theorem 3.1] and [4, Theorem 1] does not apply; in particular, we do not know in general whether a $T^{\infty}$-limit over $\mathcal{R}$ is definable in $\mathcal{P}(\mathcal{R})$.

(4) If $W_{\iota}=W_{1}$ for all $\iota$, then $K$ is a pfaffian limit over $\mathcal{R}$ as introduced in [5, Definition 4.4].

\section{Towards the proof of Theorem A}

Let $M \subseteq \mathbb{R}^{n}$ be a definable $C^{2}$-manifold of dimension $m$.

\section{Pfaffian fiber cutting}

We fix a finite family $\Delta=\left\{d^{1}, \ldots, d^{q}\right\}$ of definable nested distributions on $M$; we write $d^{p}=\left(d_{0}^{p}, \ldots, d_{k(p)}^{p}\right)$ for $p=1, \ldots, q$. As in [5, Section 3], we associate to $\Delta$ the following set of distributions on $M$ :

$$
\mathcal{D}_{\Delta}:=\left\{d_{0}^{0} \cap d_{k(1)}^{1} \cap \cdots \cap d_{k(p-1)}^{p-1} \cap d_{j}^{p}: p=1, \ldots, q \text { and } j=0, \ldots, k(p)\right\},
$$

where we put $d_{0}^{0}:=g_{M}$. If $N$ is a $C^{2}$-submanifold of $M$ compatible with $\mathcal{D}_{\Delta}$, we let $d^{\Delta, N}=\left(d_{0}^{\Delta, N}, \ldots, d_{k(\Delta, N)}^{\Delta, N}\right)$ be the nested distribution on $N$ obtained by listing the set $\left\{g^{N}: g \in \mathcal{D}_{\Delta}\right\}$ in order of decreasing dimension. In this situation, if $V_{p}$ is an integral manifold of $d_{k(p)}^{p}$, for $p=1, \ldots, q$, then the set $N \cap V_{1} \cap \cdots \cap V_{q}$ is an integral manifold of $d_{k(\Delta, N)}^{\Delta, N}$.

Let $A \subseteq M$ be definable. For $I \subseteq\{1, \ldots, q\}$ we put $\Delta(I):=\left\{d^{p}: p \in I\right\}$.

\footnotetext{
${ }^{1}$ For instance, a positive answer to this question for all $e$ definable in $\mathcal{P}(\mathcal{R})$ would imply the second part of Hilbert's 16th problem.
} 
Lemma 2.1 Let $I \subseteq\{1, \ldots, q\}$. Then there is a finite partition $\mathcal{P}$ of definable $C^{2}$-cells contained in $A$ such that $\mathcal{P}$ is compatible with $\mathcal{D}_{\Delta(J)}$ for every $J \subseteq\{1, \ldots, q\}$ and

(i) $\operatorname{dim} d_{k(\Delta(I), N)}^{\Delta(I), N}=0$ for every $N \in \mathcal{P}$;

(ii) whenever $V_{p}$ is a Rolle leaf of $d^{p}$ for $p \in I$, every component of $A \cap \bigcap_{p \in I} V_{p}$ intersects some cell in $\mathcal{P}$.

Proof By induction on $\operatorname{dim} A$; if $\operatorname{dim} A=0$, there is nothing to do, so we assume $\operatorname{dim} A>0$ and the corollary is true for lower values of $\operatorname{dim} A$. By [5, Proposition 2.2] and the inductive hypothesis, we may assume that $A$ is a $C^{2}$-cell compatible with $\mathcal{D}_{\Delta(J)}$ for $J \subseteq\{1, \ldots, q\}$. Thus, if $\operatorname{dim} d_{k(\Delta(I), A)}^{\Delta(I, A}=0$, we are done; otherwise, we let $\phi$ and $B$ be as in [5, Lemma 3.1] with $\Delta(I)$ in place of $\Delta$.

Let $V_{p}$ be a Rolle leaf of $d^{p}$ for each $p$; it suffices to show that every component of $X:=A \cap \bigcap_{p \in I} V_{p}$ intersects $B$. However, since $d_{k(\Delta(I), A)}^{\Delta(I, A}$ has dimension, $X$ is a closed, embedded submanifold of $A$. Thus, $\phi$ attains a maximum on every component of $X$, and any point in $X$ where $\phi$ attains a local maximum belongs to $B$.

Corollary 2.2 Let $d$ be a definable nested distribution on $M$ and $m \leq n$. Then there is a finite partition $\mathcal{P}$ of $C^{2}$-cells contained in $A$ such that for every Rolle leaf $V$ of $d$, we have

$$
\Pi_{m}(A \cap V)=\bigcup_{N \in \mathcal{P}} \Pi_{m}(N \cap V)
$$

and for every $N \in \mathcal{P}$, the set $N \cap V$ is a submanifold of $U, \Pi_{m} \Upsilon_{(N \cap V)}$ is an immersion and for every $n^{\prime} \leq n$ and every strictly increasing $\lambda:\left\{1, \ldots, n^{\prime}\right\} \longrightarrow\{1, \ldots, n\}$, the projection $\Pi_{\lambda} \Upsilon_{(N \cap V)}$ has constant rank.

Proof Apply Lemma 2.1 with $q:=n+1, d^{p}:=\operatorname{ker} d x_{p}$ for $p=1, \ldots, n, d^{q}:=d$ and $I:=\{1, \ldots, m, n+1\}$.

\section{$T^{\infty}$-limits}

We assume that $M$ has a definable $C^{2}$-carpeting function $\phi$, and we let $d=\left(d_{0}, \ldots, d_{k}\right)$ be a definable distribution on $M$ with core distribution $e=\left(e_{0}, \ldots, e_{l}\right)$.

First, we reformulate [5, Proposition 4.7]. We adopt the notation introduced before [5, Proposition 4.6] and note that the $q$ in [5, Remark 4.2] can be chosen independent of the particular $W$. 
Proposition 2.3 Let $\left(V_{\iota}\right)$ be a $T^{\infty}$-sequence of integral manifolds of $d$ with core sequence $\left(W_{\iota}\right)$, and assume that $K^{\prime}:=\lim _{\iota}$ fr $V_{\iota}$ exists. Then $K^{\prime}$ is a finite union of $T^{\infty}$-limits obtained from $d^{M^{\prime}}$ with core sequences among $\left(\left(W_{\iota}\right)_{1}^{M^{\prime}}\right)_{\iota}, \ldots,\left(\left(W_{\iota}\right)_{q}^{M^{\prime}}\right)_{\iota}$.

Proof Exactly as for [5, Proposition 4.7], except for replacing "core $W$ " by "core sequence $\left(W_{\iota}\right)$ " and "core $W_{p}^{M^{\prime}}$ " by "core sequence $\left(\left(W_{\iota}\right)_{p}^{M^{\prime}}\right)$ ".

Second, as we do not know yet whether $T^{\infty}$-limits are definable in an o-minimal structure, we work with the following notion of dimension (see also van den Dries and Speissegger [2, Section 8.2]): we call $N \subseteq \mathbb{R}^{n}$ a $C^{0}$-manifold of dimension $p$ if $N \neq \emptyset$ and each point of $N$ has an open neighbourhood in $N$ homeomorphic to $\mathbb{R}^{p}$; in this case $p$ is uniquely determined (by a theorem of Brouwer), and we write $p=\operatorname{dim}(N)$. Correspondingly, a set $S \subseteq \mathbb{R}^{n}$ has dimension if $S$ is a countable union of $C^{0}$-manifolds, and in this case put

$$
\operatorname{dim}(S):= \begin{cases}\max \left\{\operatorname{dim}(N): N \subseteq S \text { is a } C^{0} \text {-manifold }\right\} & \text { if } S \neq \emptyset \\ -\infty & \text { otherwise }\end{cases}
$$

It follows (by a Baire category argument) that, if $S=\bigcup_{i \in \mathbb{N}} S_{i}$ and each $S_{i}$ has dimension, then $S$ has dimension and $\operatorname{dim}(S)=\max \left\{\operatorname{dim}\left(S_{i}\right): i \in \mathbb{N}\right\}$. Thus, if $N$ is a $C^{1}$-manifold of dimension $p$, then $N$ has dimension in the sense of this definition and the two dimensions of $N$ agree.

Corollary 2.4 In the situation of [5, Lemma 1.5], the set $\lim _{\iota} V_{\iota} \backslash \lim _{\iota}$ fr $V_{\iota}$ is either empty or has dimension $p$.

Therefore, we replace [5, Lemma 4.5] by

Proposition 2.5 Let $K$ be a $T^{\infty}$-limit obtained from $d$. Then $K$ has dimension and satisfies $\operatorname{dim} K \leq \operatorname{dim} d$.

Proof Let $\left(V_{\iota}\right)$ be a $T^{\infty}$-sequence of integral manifolds of $d$ such that $K=\lim _{\iota} V_{\iota}$. We proceed by induction on $\operatorname{dim} d$. If $\operatorname{dim} d=0$, then [5, Corollary 3.3(2)] gives a uniform bound on the cardinality of $V_{\iota}$, so $K$ is finite. So assume $\operatorname{dim} d>0$ and the corollary holds for lower values of $\operatorname{dim} d$.

By [5, Proposition 2.2 and Remark 4.2], we may assume that $M$ is a definable $C^{2}$-cell; in particular, there is a definable $C^{2}$-carpeting function $\phi$ on $M$. For each $\sigma \in \Sigma_{n}$, let $M_{\sigma, 2 n}$ be as before [5, Lemma 1.3] with $d_{k}$ in place of $d$. Then by that lemma, $M=\bigcup_{\sigma \in \Sigma} M_{\sigma, 2 n}$ and each $M_{\sigma, 2 n}$ is an open subset of $M$. Hence $d$ is compatible with each $M_{\sigma, 2 n}$, and after 
passing to a subsequence if necessary, we may assume that $K_{\sigma}=\lim _{\iota}\left(V_{\iota} \cap M_{\sigma, 2 n}\right)$ exists for each $\sigma$. It follows that $K=\bigcup_{\sigma \in \Sigma_{n}} K_{\sigma}$, so by [5, Lemma 1.3(2)], after replacing $M$ with each $\sigma^{-1}\left(M_{\sigma, 2 n}\right)$, we may assume that $d_{k}$ is $2 n$-bounded. Passing to a subsequence again, we may assume that $K^{\prime}:=\lim _{\iota}$ fr $V_{\iota}$ exists as well. Then by Corollary 2.4, the set $K \backslash K^{\prime}$ is either empty or has dimension $\operatorname{dim} d$. By Proposition 2.3 and the discussion before [5, Proposition 4.6], the set $K^{\prime}$ is a finite union of $T^{\infty}$-limits obtained from a definable nested distribution $d^{\prime}$ on a definable manifold $M^{\prime}$ that satisfies $\operatorname{deg} d^{\prime} \leq \operatorname{deg} d$ and $\operatorname{dim} d^{\prime}<\operatorname{dim} d$. So $K^{\prime}$ has dimension with $\operatorname{dim} K^{\prime}<\operatorname{dim} d$ by the inductive hypothesis, and the proposition is proved.

Definition 2.6 A $T^{\infty}$-limit $K \subseteq \mathbb{R}^{n}$ obtained from $d$ is proper if $\operatorname{dim} K=\operatorname{dim} d$.

Corollary 2.7 Let $K \subseteq \mathbb{R}^{n}$ be a $T^{\infty}$-limit obtained from $d$. Then $K$ is a finite union of proper $T^{\infty}$-limits over $\mathcal{R}$ of degree at most $\operatorname{deg} d$.

Proof We proceed by induction on $\operatorname{dim} d$; as in the previous proof, we assume $\operatorname{dim} d>0$ and the corollary holds for lower values of $\operatorname{dim} d$. If $\operatorname{dim} K=\operatorname{dim} d$, we are done, so assume that $\operatorname{dim} K<\operatorname{dim} d$. Also as in the previous proof, we now reduce to the case where $d_{k}$ is $2 n$-bounded and $K^{\prime}:=\lim _{\iota}$ fr $V_{\iota}$ exists. Then Corollary 2.4 implies that $K=K^{\prime}$, so the corollary follows from Proposition 2.3 and the inductive hypothesis.

Finally, $T^{\infty}$-limits over $\mathcal{R}$ are well behaved with respect to intersecting with closed definable sets. To see this, define $\mathbf{M}:=M \times(0,1)$ and write $(x, \epsilon)$ for the typical element of $\mathbf{M}$ with $x \in M$ and $\epsilon \in(0,1)$. We consider the components of $d$ as distributions on $\mathbf{M}$ in the obvious way, and we set $\mathbf{d}_{0}:=g_{\mathbf{M}}, \mathbf{d}_{1}:=d \epsilon \uparrow_{\mathbf{M}}$ and $\mathbf{d}_{1+i}:=d_{i} \cap \mathbf{d}_{1}$ for $i=1, \ldots, k$ and put $\mathbf{d}:=\left(\mathbf{d}_{0}, \ldots, \mathbf{d}_{1+k}\right)$. Moreover, whenever $e$ is a core distribution of $d$, we similarly define a corresponding core distribution $\mathbf{e}=\left(\mathbf{e}_{0}, \ldots, \mathbf{e}_{1+l}\right)$ of $\mathbf{d}$. In this situation, for every Rolle leaf $W$ of $e$ and every $\epsilon \in(0,1)$, the set $\mathbf{W}:=W \times\{\epsilon\}$ is a Rolle leaf of $\mathbf{e}$.

Proposition 2.8 Let $K$ be a $T^{\infty}$-limit obtained from $d$, and let $C \subseteq \mathbb{R}^{n}$ be a definable closed set. Then there is a definable open subset $\mathbf{N}$ of $\mathbf{M}$ and there are $q \in \mathbb{N}$ and $T^{\infty}$-limits $K_{1}, \ldots, K_{q} \subseteq \mathbb{R}^{n+1}$ obtained from $\mathbf{d}^{\mathbf{N}}$ such that $K \cap C=\Pi_{n}\left(K_{1}\right) \cup \cdots \cup \Pi_{n}\left(K_{q}\right)$.

Sketch of proof For $\epsilon>0$ put $T(C, \epsilon):=\left\{x \in \mathbb{R}^{n}: d(x, C)<\epsilon\right\}$. Note first that $K \cap C=$ $\bigcap_{\epsilon>0}(K \cap T(C, \epsilon))$, and the latter is equal to $\lim _{\epsilon \rightarrow 0}(K \cap T(C, \epsilon))$ in the sense of [5, Definition 1.7]. Next, let $\left(V_{\iota}\right)$ be a $T^{\infty}$-sequence of integral manifolds of $d$ such that $K=$ $\lim _{\iota} V_{\iota}$. Then for every $\epsilon>0$, there is a subsequence $(\iota(\kappa))$ of $(\iota)$ such that the sequence $\left(V_{\iota(\kappa)} \cap T(C, \epsilon)\right)$ converges to some compact set $K_{\epsilon}$. Note that $K_{\epsilon} \cap T(C, \epsilon)=K \cap T(C, \epsilon)$, since $T(C, \epsilon)$ is an open set. 
Fix a sequence $\left(\epsilon_{\kappa}\right)$ of positive real numbers approaching 0 , and for each $\kappa$, choose $\iota(\kappa)$ such that $d\left(V_{\iota(\kappa)} \cap T\left(C, \epsilon_{\kappa}\right), K_{\epsilon_{\kappa}}\right)<\epsilon_{\kappa}$. Passing to a subsequence if necessary, we may assume that $\lim _{\kappa} K_{\epsilon_{\kappa}}$ and $\lim _{\kappa}\left(V_{\iota(\kappa)} \cap T\left(C, \epsilon_{\kappa}\right)\right)$ exist; note that these limits are then equal. Hence by the above,

$$
\begin{aligned}
K \cap C & =\lim _{\kappa}\left(K \cap T\left(C, \epsilon_{\kappa}\right)\right)=\lim _{\kappa}\left(K_{\epsilon_{\kappa}} \cap T\left(C, \epsilon_{\kappa}\right)\right) \\
& \subseteq \lim _{\kappa} K_{\epsilon_{\kappa}}=\lim _{\kappa}\left(V_{\iota(\kappa)} \cap T\left(C, \epsilon_{\kappa}\right)\right) .
\end{aligned}
$$

The reverse inclusion is obvious, so $K \cap C=\lim _{\kappa}\left(V_{\iota(\kappa)} \cap T\left(C, \epsilon_{\kappa}\right)\right)$. Therefore, put $\mathbf{N}:=\{(x, \epsilon) \in \mathbf{M}: d(x, C)<\epsilon\}$; then $\mathbf{N}$ is an open, definable subset of $\mathbf{M}$ and by the above $K \cap C=\lim _{\kappa}\left(V_{\iota(\kappa)} \cap \mathbf{N}^{\epsilon_{\kappa}}\right)$, where $\mathbf{N}^{\epsilon}:=\{x \in M:(x, \epsilon) \in \mathbf{N}\}$. Hence $K \cap C=$ $\lim _{\kappa} \Pi_{n}\left(\left(V_{\iota(\kappa)} \times\left\{\epsilon_{\kappa}\right\}\right) \cap \mathbf{N}\right)$. Since $\lim _{\kappa} \epsilon_{\kappa}=0$, it follows that $K \cap C=\Pi_{n}\left(\lim _{\kappa}\left(\left(V_{\iota(\kappa)} \times\right.\right.\right.$ $\left.\left.\left.\left\{\epsilon_{\kappa}\right\}\right) \cap \mathbf{N}\right)\right)$. Since the sequence $\left(V_{\iota(\kappa)} \times\left\{\epsilon_{\kappa}\right\}\right)$ is a $T^{\infty}$-sequence of integral manifolds of d, the proposition now follows from [5, Remark 4.2].

Remark 2.9 Let $\mathcal{B}$ and $\mathcal{C}$ be two definable families of closed subsets of $\mathbb{R}^{n}$. Then the $T^{\infty}$ limits in the previous proposition depend uniformly on $C \in \mathcal{C}$, for all $T^{\infty}$-limits obtained from $d$ with definable part $\mathcal{B}$. That is, there are $\mu, q \in \mathbb{N}$, a bounded, definable manifold $\mathbf{M} \subseteq \mathbb{R}^{n+\mu+1}$, a definable nested distribution $\mathbf{d}$ on $\mathbf{M}$ and a definable family $\mathbf{B}$ of subsets of $\mathbb{R}^{n+\nu+1}$ such that whenever $K$ is a $T^{\infty}$-limit obtained from $d$ with definable part $\mathcal{B}$ and $C \in \mathcal{C}$, there are $T^{\infty}$-limits $K_{1}, \ldots, K_{q} \subseteq \mathbb{R}^{n+\nu+1}$ obtained from $\mathbf{d}$ with definable part $\mathbf{B}$ such that $K \cap C=\Pi_{n}\left(K_{1}\right) \cup \cdots \cup \Pi_{n}\left(K_{q}\right)$.

\section{O-minimality and proof of Theorem A}

Similar to $[3,7]$, we show that all sets definable in $T^{\infty}(\mathcal{R})$ are of the following form:

Definition 3.1 A set $X \subseteq \mathbb{R}^{m}$ is a basic $T^{\infty}$-set if there exist $n \geq m$, a definable, bounded $C^{2}$-manifold $M \subseteq \mathbb{R}^{n}$, a definable nested distribution $d$ on $M$ with core distribution $e$ and, for $\kappa \in \mathbb{N}$, a $T^{\infty}$-sequence $\left(V_{\kappa, \iota}\right)_{\iota}$ of integral manifolds of $d$ with core sequence $\left(W_{\kappa, \iota}\right)_{\iota}$ corresponding to $e$ and definable part $\mathcal{B}$ independent of $\kappa$, such that:

(i) for each $\kappa$, the limit $K_{\kappa}:=\lim _{\iota} V_{\kappa, \iota}$ exists in $\mathcal{K}_{n}$;

(ii) the sequence $\left(\Pi_{m}\left(K_{\kappa}\right)\right)_{\kappa}$ is increasing and has union $X$.

In this situation, we say that $X$ is obtained from $d$ with core distribution $e$ and definable part $\mathcal{B}$. A $T^{\infty}$-set is a finite union of basic $T^{\infty}$-sets. We denote by $T_{m}^{\infty}$ the collection of all $T^{\infty}$-sets in $\mathbb{R}^{m}$ and put $T^{\infty}:=\left(T_{m}^{\infty}\right)_{m \in \mathbb{N}}$. 
Proposition 3.2 In the situation of Definition 3.1, there is an $N \in \mathbb{N}$ such that every basic $T^{\infty}$-set obtained from $d$ with core distribution $e$ and definable part $\mathcal{B}$ has at most $N$ components. In particular, if $X \subseteq \mathbb{R}^{m}$ is a $T^{\infty}$-set and $l \leq m$, there is an $N \in \mathbb{N}$ such that for every $a \in \mathbb{R}^{l}$ the fiber $X_{a}$ has at most $N$ components.

Proof Let $N$ be a bound on the number of components of the sets $W \cap B$ as $W$ ranges over all Rolle leaves of $e$ and $B$ ranges over $\mathcal{B}$. Let $X$ be a basic $T^{\infty}$-set as in Definition 3.1. Then each $V_{\kappa, \iota}$ has at most $N$ components, so each $K_{\kappa}$ has at most $N$ components, and hence $X$ has at most $N$ components. Combining this observation with Remark 2.9 yields, for every $T^{\infty}$-set $X \subseteq \mathbb{R}^{m}$, a uniform bound on the number of connected components of the fibers of $X$.

Proposition 3.3 (1) Any coordinate projection of a $T^{\infty}$-limit over $\mathcal{R}$ is a $T^{\infty}$-set.

(2) Every bounded definable set is a $T^{\infty}$-set.

(3) Let $d$ be a definable nested distribution on $M:=(-1,1)^{n}$ and $L$ be a Rolle leaf of d. Then $L$ is a $T^{\infty}$-set.

Proof (1) is obvious. For (2), let $C \subseteq \mathbb{R}^{n}$ be a bounded, definable cell. By cell decomposition, it suffices to show that $C$ is a $T^{\infty}$-set. Let $\phi$ be a definable carpeting function on $C$. Then $C=\bigcup_{i=1}^{\infty} \operatorname{cl}\left(\phi^{-1}((1 / i, \infty))\right)$, so let $\mathbf{C}:=\{(x, r) \in C \times(0,1): \phi(x)>r\}$ and put $\mathbf{d}_{1}:=\operatorname{ker} d r \Gamma_{\mathbf{C}}$ and $\mathbf{d}:=\left(g_{\mathbf{C}}, \mathbf{d}_{1}\right)$. Then for $r>0$, the set $\mathbf{C}^{r}=\phi^{-1}((r, \infty)) \times\{r\}$ is an admissible integral manifold of $\mathbf{d}$ with core $\mathbf{C}$ and definable part $\mathbf{C}^{r}, \operatorname{so~} \operatorname{cl}\left(\mathbf{C}^{r}\right)$ is a $T^{\infty}$-limit obtained from $\mathbf{d}$.

(3) Let $\phi$ be a carpeting function on $M$. Then

$$
L=\bigcup_{i=1}^{\infty} \operatorname{cl}\left(L \cap \phi^{-1}((1 / i, \infty))\right),
$$

so we let $\mathbf{M}:=\{(x, r) \in M \times(0,1): \phi(x)>r\}$ and put $\mathbf{d}_{0}:=g_{\mathbf{M}}, \mathbf{d}_{1}:=\operatorname{ker} d r \uparrow_{\mathbf{M}}$, $\mathbf{d}_{1+i}:=\mathbf{d}_{1} \cap d_{i}$ for $i=1, \ldots, k$ and $\mathbf{d}:=\left(\mathbf{d}_{0}, \ldots, \mathbf{d}_{1+k}\right)$. Let $L_{1}, \ldots, L_{q}$ be the components of $(L \times(0,1)) \cap \mathbf{M}$; note that each $L_{p}$ is a Rolle leaf of $\mathbf{d}$. Thus for $r>0$ and each $p$, the set $L_{p} \cap \phi^{-1}((r, \infty))$ is an admissible integral manifold of $\mathbf{d}$ with core $L_{p}$ and definable part $\mathbf{M}^{r}=\phi^{-1}((r, \infty)) \times\{r\}$.

Proposition 3.4 The collection of all $T^{\infty}$-sets is closed under taking finite unions, finite intersections, coordinate projections, cartesian products, permutations of coordinates and topological closure. 
Proof Closure under taking finite unions, coordinate projections and permutations of coordinates is obvious from the definition and the properties of nested pfaffian sets over $\mathcal{R}$.

For topological closure, let $X \subseteq \mathbb{R}^{m}$ be a basic $T^{\infty}$-set with associated data as in Definition 3.1. Then

$$
\operatorname{cl}(X)=\lim _{\kappa} \Pi_{m}\left(K_{\kappa}\right)=\Pi_{m}\left(\lim _{\kappa} \lim _{\iota} V_{\kappa, \iota}\right)=\Pi_{m}\left(\lim _{\kappa} V_{\kappa, \iota(\kappa)}\right)
$$

for some subsequence $(\iota(\kappa))_{\kappa}$, so $\operatorname{cl}(X)$ is a $T^{\infty}$-set by Proposition 3.3(1).

For cartesian products, let $X_{1} \subseteq \mathbb{R}^{m_{1}}$ and $X_{2} \subseteq \mathbb{R}^{m_{2}}$ be basic $T^{\infty}$-sets, and let $M^{i} \subseteq \mathbb{R}^{n_{i}}$, $d^{i}=\left(d_{0}^{i}, \ldots, d_{k^{i}}^{i}\right), e^{i}=\left(e_{0}^{i}, \ldots, e_{l^{i}}^{i}\right)$ and $\left(V_{\iota, \kappa}^{i}\right)$ be the data associated to $X_{i}$ as in Definition 3.1, for $i=1,2$. We assume that both $M^{1}$ and $M^{2}$ are connected; the general case is easily reduced to this situation. Define

$$
\mathbf{M}:=\left\{(x, y, u, v):(x, u) \in M^{1} \text { and }(y, v) \in M^{2}\right\},
$$

where $x$ ranges over $\mathbb{R}^{m_{1}}, y$ over $\mathbb{R}^{m_{2}}, u$ over $\mathbb{R}^{n_{1}-m_{1}}$ and $v$ over $\mathbb{R}^{n_{2}-m_{2}}$. We interpret $d^{i}$ and $e^{i}$ as sets of distributions on $\mathbf{M}$ correspondingly, for $i=1,2$, and we define $\mathbf{d}:=\left(d_{0}^{1}, \ldots, d_{k^{1}}^{1}, d_{k^{1}}^{1} \cap d_{1}^{2}, \ldots, d_{k^{1}}^{1} \cap d_{k^{2}}^{2}\right)$ and $\mathbf{e}:=\left(e_{0}^{1}, \ldots, e_{l^{1}}^{1}, e_{l^{1}}^{1} \cap e_{1}^{2}, \ldots, e_{l^{1}}^{1} \cap e_{l^{2}}^{2}\right)$. Since $M^{1}$ and $M^{2}$ are connected, each set

$$
V_{\kappa, \iota}:=\left\{(x, y, u, v):(x, u) \in V_{\kappa, \iota}^{1} \text { and }(y, v) \in V_{\kappa, \iota}^{2}\right\}
$$

is an admissible integral manifold of $\mathbf{d}$ with core distribution $\mathbf{e}$. It is now easy to see that for each $\kappa$, the limit $K_{\kappa}:=\lim _{\iota} V_{\kappa, \iota}$ exists in $\mathcal{K}_{n_{1}+n_{2}}$, and that the sequence $\left(\Pi_{k_{1}+k_{2}}\left(K_{\kappa}\right)\right)$ is increasing and has union $X_{1} \times X_{2}$.

For intersections, let $X_{1}, X_{2} \subseteq \mathbb{R}^{m}$ be basic $T^{\infty}$-sets. Then $X_{1} \cap X_{2}=\Pi_{k}\left(\left(X_{1} \times X_{2}\right) \cap \Delta\right)$, where $\Delta:=\left\{(x, y) \in \mathbb{R}^{m} \times \mathbb{R}^{m}: x_{i}=y_{i}\right.$ for $\left.i=1, \ldots, m\right\}$. Therefore, we let $X \subseteq \mathbb{R}^{m}$ be a basic $T^{\infty}$-set and $C \subseteq \mathbb{R}^{m}$ be closed and definable, and we show that $X \cap C$ is a $T^{\infty}$-set. Let the data associated to $X$ be as in Definition 3.1, and let $\mathbf{M}$, $\mathbf{d}$ and $\mathbf{e}$ be associated to that data as before Proposition 2.8. Let also $\mathbf{N}$ be the open subset of $\mathbf{M}$ given by that proposition with $C^{\prime}:=C \times \mathbb{R}^{n-m}$ in place of $C$. Then by that proposition, there is a $q \in \mathbb{N}$ such that for every $\kappa$ the set $K_{\kappa} \cap C^{\prime}$ is the union of the projections of $T^{\infty}$-limits $K_{\kappa}^{1}, \ldots, K_{\kappa}^{q}$ obtained from $\mathbf{d}^{\mathbf{N}}$. Note that each $K_{\kappa}^{j}$ is the limit of a $T^{\infty}$-sequence of integral manifolds of $\mathbf{d}^{\mathbf{N}}$ with core distribution $\mathbf{e}^{\mathbf{N}}$. Replacing each sequence $\left(K_{\kappa}^{j}\right)$ by a (possibly finite) subsequence if necessary, we may assume that each sequence $\left(\Pi_{m}\left(K_{\kappa}^{j}\right)\right)$ is increasing. Then each $X_{j}:=\bigcup_{\kappa} K_{\kappa}^{j}$ is a basic $T^{\infty}$-set and $X \cap C=X_{1} \cup \cdots \cup X_{q}$.

Proposition 3.5 Let $X \subseteq \mathbb{R}^{m}$ be a $T^{\infty}$-set. Then $\operatorname{bd}(X)$ is contained in a closed $T^{\infty}$-set with empty interior. 
Proof Let the data associated to $X$ be given as in Definition 3.1 and write $d=\left(d_{0}, \ldots, d_{k}\right)$. Note that

$$
\operatorname{bd}(X) \subseteq \lim _{\kappa} \operatorname{bd}\left(\Pi_{m}\left(K_{\kappa}\right)\right) .
$$

Fix an arbitrary $\kappa$; since $\Pi_{m}\left(K_{\kappa}\right)=\lim _{\iota} \Pi_{m}\left(V_{\kappa, \iota}\right)$ we may assume, by Corollary 2.2, [5, Remark 4.2] and after replacing $M$ if necessary, that $\left.\Pi_{k}\right|_{d_{k}}$ is an immersion and has constant rank $r \leq m$; in particular, $\operatorname{dim}\left(V_{\kappa, L}\right) \leq m$. If $r<m$, then each $\Pi_{m}\left(K_{\kappa}\right)$ has empty interior by Proposition 2.4 , so

$$
\lim _{\kappa} \operatorname{bd}\left(\Pi_{m}\left(K_{\kappa}\right)\right)=\lim _{\kappa} \Pi_{m}\left(K_{\kappa}\right)=\Pi_{m}\left(\lim _{\kappa} K_{\kappa}\right)=\Pi_{m}\left(\lim _{\kappa} V_{\kappa, \iota(\kappa)}\right)
$$

for some subsequence $(\iota(\kappa))$, and we conclude by Propositions 2.5 and 3.3(1) in this case. So assume that $r=m$; in particular, $\Pi_{m}\left(V_{\kappa, \iota}\right)$ is open for every $\kappa$ and $\iota$. In this case, since $M$ is bounded, we have $\operatorname{bd}\left(\Pi_{m}\left(K_{\kappa}\right)\right) \subseteq \Pi_{m}\left(\lim _{\iota}\right.$ fr $\left.V_{\kappa, \iota}\right)$ for each $\kappa$. Hence

$$
\lim _{\kappa} \operatorname{bd}\left(\Pi_{m}\left(K_{\kappa}\right)\right) \subseteq \Pi_{m}\left(\lim _{\kappa} \lim _{\iota} \operatorname{fr} V_{\kappa, L}\right)=\Pi_{m}\left(\lim _{\kappa} \operatorname{fr} V_{\kappa, \iota(\kappa)}\right)
$$

for some subsequence $(\iota(\kappa))$. Now use Propositions 2.3 and 3.3(1).

Following [8] and [3], and proceeding exactly as in [7, Corollary 3.11 and Proposition 3.12] using the previous propositions, we obtain:

Proposition 3.6 (1) Let $X \subseteq \mathbb{R}^{m}$ be a $T^{\infty}$-set, and let $1 \leq l \leq m$. Then the set $B:=\left\{a \in \mathbb{R}^{l}: \operatorname{cl}\left(X_{a}\right) \neq \operatorname{cl}(X)_{a}\right\}$ has empty interior.

(2) Let $X \subseteq[-1,1]^{m}$ be a $T^{\infty}$-set. Then $[-1,1]^{m} \backslash X$ is also a $T^{\infty}$-set.

For $m \in \mathbb{N}$, let $\mathcal{T}_{m}$ be the collection of all $T^{\infty}$-sets $X \subseteq I^{m}$.

Corollary 3.7 The collection $\mathcal{T}:=\left(\mathcal{T}_{m}\right)_{m \in \mathbb{N}}$ forms an o-minimal structure on $I$.

Proof of Theorem A For each $m$, let $\tau_{m}: \mathbb{R}^{m} \longrightarrow(-1,1)^{m}$ be the (definable) homeomorphism given by

$$
\tau_{m}\left(x_{1}, \ldots, x_{m}\right):=\left(\frac{x_{1}}{1+x_{1}^{2}}, \ldots, \frac{x_{m}}{1+x_{m}^{2}}\right),
$$

and let $\mathcal{S}_{m}$ be the collection of sets $\tau_{m}^{-1}(X)$ with $X \in \mathcal{T}_{m}$. By Corollary 3.7, the collection $\mathcal{S}=\mathcal{S}:=\left(\mathcal{S}_{m}\right)_{m}$ gives rise to an o-minimal expansion $T^{\infty}(\mathcal{R})$ of $\mathcal{R}$. By Proposition 3.3(2), every definable set is definable in $T^{\infty}(\mathcal{R})$. But if $L$ is a Rolle leaf of a definable nested distribution $d$ on $\mathbb{R}^{n}$, then $\tau_{n}(L)$ is a Rolle leaf of the pullback $\left(\tau_{n}^{-1}\right)^{*} d$. It follows from Proposition 3.3(3) that $\tau_{n}(L) \in \mathcal{T}_{n}$, so $L$ is definable in $T^{\infty}(\mathcal{R})$. Therefore, $\mathcal{N}(\mathcal{R})$ is a reduct of $T^{\infty}(\mathcal{R})$ in the sense of definability. 


\section{Proof of Theorem B}

First, we establish [5, Proposition 7.1] with " $T^{\infty}$-limit" and " $T^{\infty}(\mathcal{R})$ " in place of "pfaffian limit" and " $\mathcal{P}(\mathcal{R})$ ". To do so, we proceed exactly as in [5], making the following additional changes.

(B1) Replacing "admissible sequence" with " $T^{\infty}$-sequence", we obtain corresponding versions of Lemma 4.8, Remark 4.9, Proposition 4.11, Corollary 4.13 and Proposition 5.3 in [5].

(B2) Using (B1), we obtain the corresponding version of [5, Proposition 7.1].

Second, assuming that $\mathcal{R}$ admits analytic cell decomposition, (B2) and [5, Proposition 10.4] imply that every $T^{\infty}$-limit over $\mathcal{R}$ is definable in $\mathcal{N}(\mathcal{R})$; in particular, $T^{\infty}(\mathcal{R})$ and $\mathcal{N}(\mathcal{R})$ are interdefinable. Hence, by [5, Corollary 1], $T^{\infty}(\mathcal{R})$ and $\mathcal{P}(\mathcal{R})$ are interdefinable. Replacing once more $\mathcal{R}$ by $\mathcal{P}(\mathcal{R})$, Theorem B is now proved.

\section{References}

[1] L van den Dries, Limit sets in o-minimal structures, from: "Proceedings of the RAAG Summer School Lisbon 2003: O-minimal structures", (M Edmundo, D Richardson, A J Wilkie, editors) (2005) 172-215

[2] L van den Dries, P Speissegger, The real field with convergent generalized power series, Trans. Amer. Math. Soc. 350 (1998) 4377-4421

[3] J-M Lion, J-P Rolin, Volumes, feuilles de Rolle de feuilletages analytiques et théorème de Wilkie, Ann. Fac. Sci. Toulouse Math. (6) 7 (1998) 93-112

[4] J-M Lion, P Speissegger, A geometric proof of the definability of Hausdorff limits, Selecta Math. (N.S.) 10 (2004) 377-390

[5] J-M Lion, P Speissegger, The theorem of the complement for nested sub-pfaffian sets, Duke Math. J. 155 (2010) 35-90

[6] D Marker, C I Steinhorn, Definable types in o-minimal theories, J. Symbolic Logic 59 (1994) 185-198

[7] P Speissegger, The Pfaffian closure of an o-minimal structure, J. Reine Angew. Math. 508 (1999) 189-211

[8] A J Wilkie, A theorem of the complement and some new o-minimal structures, Selecta Math. (N.S.) 5 (1999) 397-421 
IRMAR, Université de Rennes I, Campus de Beaulieu, 35042 Rennes cedex, France

Department of Mathematics \& Statistics, McMaster University, 1280 Main Street West, Hamilton, Ontario L8S 4K1, Canada

jean-marie.lion@univ-rennes1.fr, speisseg@math.mcmaster.ca 